the second case, reported by Mr. J. Knowsley 'Thornton, ${ }^{2}$ the symptoms of acute biliary colic were present; they recurred once and were then followed by jaundice and choluria. Here the gall-bladder contained a number of hydatids and much bile-stained fluid, the cystic duct being patent, and one other cyst was removed from the omentum. "The third case is reported by Mr. Frederick Page," the symptoms being chiefly vomiting and epigastric pain. A tumour was felt and on aspiration yielded perfectly colourless fluid. Cholecystotomy was performed and the organ was found to contain a single cyst without daughter cysts, the cystic duct being patent. Mr. A. W. Mayo Robson ${ }^{+}$mentions a case of hydatid disease simulating gall-stone attacks in which hydatids appear to have been discharged from the liver into the bile-ducts, infective cholangitis being the result. But here the disease appears to have originated in the liver. The same author draws attention to certain cases, all of which appear either to have originated in the liver or to have been complicated by communications between the liver and gall-bladder, leaving the original site of the disease in doubt.

With regard to the diagnosis of hydatid disease of the gall-bladder I am unaware of any case having as yet been diagnosed before operation. The difficulties in the way of making such a diagnosis are obvious and beyond the recognition of a tumour as being the gall-bladder in a state of distension (presuming always that no other swellings exist), I doubt if a definite diagnosis is possible. As to the treatment of such cases it must necessarily be considered from the special aspect of each individual case. In my own case cholecystectomy would have been clearly indicated (the cystic duct being no longer patent and the mucous membrane completely destroyed by pressure) even bad the case not been considered one of malignant disease. The patient, I may say, has made an uneventful recovery. The specimen has been deposited in the Hunterian Museum, where it is at present the only one of its kind.

Montagu-street, $\mathrm{W}$.

\section{TETANUS FOLLOWING REVACCINATION ON THE LEG;}

RECOVERY AF'TER PROLONGED ADMINISTRATION OF CHLORAL HYDRATE.'

BY WILLIAM FINDLAY, M.D. GLASG., AND

JOHN WAINMAN FINDLAY, M.D. GLASG.,

ASSISTANT PHYSICIAN, GLASGOW ROYAL INFIRMARY, AND ASSISTANT TO THE PROFESSOR OF MEDICINE, ST. MUNGO'S COLLEGE, GLASGOW.

THE patient, a female, aged 21 years, was revaccinated on March 8th, 1901. As she desired very particularly to use her arms in a fortnight's time she asked that the inoculation should be done on the leg. Accorảingly a mark, a little over half a square inch in size, was made on the outer side of the right leg, about midway between the knee and the ankle. Calf lymph, as supplied by the Health Office of the City of Glasgow, was used, and more than the usual precautions were taken to prevent septic infection. The middle third of the leg was cleaned with turpentine and then with methylated spirits, after which the part was again rubbed over with a carbolic solution ( 1 in 40). The lancet used was always kept in a glass of carbolic solution. A large adhesive thick felt or bunion plaster was then applied around the vaccination mark and was kept in position by a strip of adhesive planter, the object being to prevent any contact of stocking or clnthes with the wound on the way from the surgery to her home antil it was quite dry. The vaccinatiın "took," constitutional and local symptoms were quite insignificant, and the patient was able throughout to do serious mental work and to go about as though nothing had happened.

On the 18th (10 days after inoculation of the vaccine) she attended a concert in a hall which is notorious for itdraughts and she occupied a seat in a specially draughty place. While listening to the music she complained to her

2 The Lancet April 4th. 1891, p. 763.

3 Tre LanceT. April 9th. 1898, p 995.

4 Diseases of the Gall-bladder and Bile-ructs. pp 42, 43

1 A paper reart at a meeting of the Glasgow Eastern Medical Society, held on Feb. 5 th, 1902 . friends of the discomfort she was experiencing trom the draught. On the 20th (12 days after inoculation) she first complained of pain in her back, ielt out of sorts, and could not settle to work. On the 21st she remained in bed and was first seen by one of us. Her temperature was normal; the pulse was 80 , small, of somewhat high tension, and regular, and over and above the pain in the back there was complaint of stiffness of the neck and the jaws. There was no sorethroat and no abnormality of the thoracic or abdominal organs was detected. Through some misapprehension the adhesive plaster had never been removed from the leg, and when crust and plaster were pulled off the vaccination mark did not look particularly healthy ; it was covered by a greyish-coloured slough and there was a slight discharge of thin dirty yellow pus. There was, however, no surrounding inflammation of the skin and no enlargement or tenderness of the popliteal or femoral glands. A bread poultice was ordered and this at once converted the foul sore into a healthy, healing wound, which was afterwards dressed daily with moist boric lint. (We may as well state here and be done with it that in three weeks' time from the inoculation the vaccination sore was healed.) Under the impression that she was possibly suffering from a rheumatic affection of the muscles about the neck salicylate of soda (15 grains), four times daily, was prescribed.

On the morning of the 22nd her condition was much the same as on the previous night. As the day wore on, however, the jaws got stiffer and gradually closed till by the evening she could only open her mouth to about half its usual extent. There was no rigidity or spasm of the masseters or depressors of the jaw to be detected, and with a little trouble we could still assure ourselves that there was no sore-throat. There was no diffculty in swallowing. The head was very distinctly retracted, and though the patient could raise it off the pillow and make the chin touch the sternum, she complained much of stiffness and slightly of pain in the back of the neck. The muscles of the back of the neck, however, did not feel specially rigid. Slight tenseness of the muscles of the abdomen was felt, but there were no spasms of these muscles and no pain was complained of in them. No hysterical stigmata were present. The temperature was normal and the pulse was slow (68) and regular. On the morning of the 23rd the patient seemed much better, though she had not slept much during the night She could or en the jaws well and was quite happy about herself. As the bowels had not moved since the 20 th, though cascara sagrada had been given on the 22nd, the same drug, in tabloid form. was again ordered, to be followed by an enema. In the evening the jaws could be less widely opened. Pain and, for the first time, twitching of the muscles of the abdomen in the region of the right groin were complained of, and also some stiffness in the right leg. The left knee-jerk was active, and on the right side it was markedly exaggerated, showing a tendency to clonus. There was no disturbance of sensation. The salicylate of soda was ordered to be stopped and chloral hydrate, in 20-grain doses, to be given every two or three hours till the spasm was relieved and sleep was induced.

On the 24th it was ascertained that in spite of the administration of 60 grains of chloral hydrate the patient had had very little sleep during the night. In the morning the jaws could be opened sufficiently to give exit to the protruded tongue, but no more. The abdomen felt hard and board-like from constant tonic contraction of the muscles, and every now and then spasms or paroxysmal contractions occurred. The spasmodic contraction of the abdominal muscles was more marked over the right iliac fossa and caused con-iderable pain, which made her cry out. The spasm in the neck muscler was not constant, but was absent for short periods, and it varied in intensity from time to time. She could move the head backwards and forwards and from side to side within a limited range, but movement was carried out slewly with both pain and difficulty. There was some trouble in passing urine, but no actual retention. No chloral was given in the morning, and by the afternoon it was noticed that the patient had a fixed expression about the face, a- if the had suddenly become aged. The eyes had a peeping look with small palpebral fi-sure, and curuld not be fully opened wing to the contraction of the orbiculares palpehrarum. The naso-labial and mouth angle folds were deeper than was normal, and the orbicularis oris and buccinators seemed and 
felt tense. The abdominal muscles were still hard and board-like and paroxysmal spasms were occurring in the right iliac fossa. There were pain and stiffness in the back, and at times we thought that we could make out slight arching of the same. The head was distinctly retracted, and only with great difficulty and some pain could the chin be made to come near, but not to touch, the sternum. 'The extensor muscles of the neck were very hard and tense and, for the first time since the illness began, the masseter mu:cles were felt to stand out as stout bands. The legs were kept fully extended, and the feet were so strongly flexed as almost to continue the straight line of the legs. By gentle movement, however, after some little time this tonic spasm of the calf muscles could be overcome, and the feet could be brought forward almont to a right angle with the legs, but they would come no further. The patient distinctly objected to have her knee and hip-joints flexed and she could not sit up in bed. She had had 20 grains of chloral hydrate twice during the early part of the afternoon.

Up to this point, though tetanus had never been absent from our minds, it had seemed to us that all the symptoms could be explained by a diagno-is of hysteria ; and the varying degree of the muscular rigiditythe malady appearing at one time to be progressing and at another to be receding-and the fact that such rigidity by gentle manipulation could to a great extent be overcome, coupled with the distinctly neurotic character of the patient, in spite of the absence of definite hysterical stigmata, seemed to lead in this direction. But now we could no longer avoid making the diagnosis of tetanu:, cerebro-spinal meningitis being exclured by the absence of fever, headache, vomiting, \&c. In the evening Professor T, $K$. Monro saw the case in consultation and agreed with us that the malady was tetanus. It was arranged to give a further dose of purgative medicine (half an ounce of castor oil) thoroughly to clear the bowel and to continue the chloral hydrate in somewhat larger doses-from 20 to 30 grains every four hours. In view of the gravity of the disease, however, it was decided to obtain a surgical opinion to consult as to the advisability of operative interference. Professor Monro made an ophthalmo:copic examination and found the fundi normal. Profe-sor Sir Hector Cameron saw the case on the evening of the 25th, when the state of the patient was somewhat better than it had been on the previous day owing to two and a half drachms of chloral having been given during the last 24 hours. Still, all the facts which we have mentioned were present. Sir Hector Cameron agreed with the diagno-is of tetanus, but did not think that it was either necessary or desirable to excise the vaccination wound or yet to give tetanus antitoxin. He advised that the chloral be continned and even pu hed, and, drawing fiom his great store of clinical experience, confidently asserted that the patient would ultimately get well.

From the 25th to the 26th the patient had had 240 grains of chloral during the 24 hours and by the 27 th 180 grains more had been given. She was under the influence of the drug and was very silly and stupidlike a half-drunken person. The eye; however, were more widely opened, there was an absence of the ominous lines ab ut the nose and mouth, and the -light rardonic grin was no longer perceptible. She could nud her head There was no spasm in the abdominal mu-cles, and the legs, in place of being extended as formerly, were now drawn up in bed. The only drawbark to her splendid feeling of bien-être was that retention of urine had cccurred. A catheter was paised at night. The improvement still continued and nn the 29th the mouth conld be more widely opened. During the lant 48 hours 120 grains of chloral had heen sufficient to allay any tendency to spasm. The catheter was passed twice during this and the preceding day. The temperature, which had previously always been nornal, was $99.0^{\circ} \mathrm{F}$. in the morning and $996^{\circ}$ at night On the $30 \mathrm{th}$ she passel urine herself, but the calls to do s.) became very frequent, and there was stinging pain at the ent of each act of micturition. The urine was faintly alktine and contained a slight sediment of pus. The tempertare was $998^{\circ}$ in the morning and $100.6^{\circ}$ at night.

Erom this point onward to the end of April there was 'ight fever of an intermitient character. the temperature reing off and on ahout $n$ rmal in the morning and never higher than $101^{\circ}$ at night The abnorm. I temuerature cincided with the applearance of the cy-titis, which persi,teil-a, shown by pus in the urine-well on till the end of July. The bladder inflammation, due, no doubt, to the catheterisation, was treated all along with salol and urotropine, but chiefly the latter, which, on the whole, acted most beneficially. The chloral, in 15-grain doses, was continued at about the rate of five grains every hour, the patient calling for it as she was in dread of the spasms returning with their old force. During the next three days advantage was taken of this improvement to wean her from the chloral and to substitute bromide of potassium, but the spasms in the abdomen and the legs returned necessitating a re-administration of the drug. From April 6th to the 22nd the spasms were kept under by the continued administration of the chloral day and night. So long as the patient was under the influence of the drug the spa-ms in the legs, the abdomen, and the back were fairly quiet and the rigidity of the muscles of the face and neck was well relaxed. Though dosed with the drug she lay in constant terror of the bed or herself being suddenly touched or jerked lest the old twitchings should come back. If anyone incautiously entered the room or walked roughly across the floor the spasms accurred. During this period she took plenty of nourishment of an entirely "sloppy" character as she was unable to chew owing to the rigidity of the jaws. By this date (A pril 22nd) she had consumed in all seven ounces three drachms and 10 grains of chloral. During the next fortnight the chloral was gradually le isened-exactly an ounce was given-until it was stopued altogether, and bromide of potassium in 15-grain doses four times daily was again substituted for a week, after which she was put on iron. Thus during her entire illness she took a little over eight ounces of chloral, or, to be particular, exactly eight ounces and three drachms, plus 10 grains, and never at any time was there the least sign of cardiac failure.

Towards the end of the first week in May, a day or two after the chlorai was withheld, the nurse began to encourage the patient to sit up in bed, and later to get out of bed, but it was slow work, as the spaims threatened to ref urn on the least exertion, and her extremely weak and. nervous condition seemed, throngh terror, to anticipate, if not actually to make, them. The knees and hips remained extended and for some time she could not sit down in a chair properly, but had to be propped up like a stiffened lay figure; and later, when she tried to walk, even though supported on each side, she could not lift or flex her limbs. In the legs the -pasm was la.t to leave the flexors of the ankles; she could not set her feet down flat on the ground and walked for a period on her toes. Perhaps fear of the spasms returning had something to do with her extreme helple-sness and slow recovery. By perseverance, however, she gradually gained confidence and p.wer, and towards the end of the month was walking with the assistance of the nurse's arm and a stick, though still holding her neck and back very stiffly. The tri-mus al-o remained to a slight extent and was the last of the rigidities to disappear. Solid food could now be taken in place of the finely-chopped meats required in the earlier part of the month. She was also regaining her natural sleep at night which was uncertain after the loss of the chloral. Early in June she went to the coast, where she was golfing by the end of the month and, needless to say, free from all tetanic symptoms. It should have been mentirned that the muscles of the arms were never at any time affected and constipation was a marked feature throughout the entire illness.

\section{CONSIDERATIONS AND CONCLUSIONS.}

In spite of the fact that we have no bacteriological evidence to offer, it cannot be cloubted that the above was a true, though by no means a classical, case of tetanus. Further, as already mentioned, the patient was seen by Profes-or sir Hect r O. Cameron and Professor T. K. Monro, and both concurred in this diagnosiv. That we were not dealing with a simple rheumati-m occasioned by the draught in which the patient sat two days before symptoms showed them-elves was made evident by the stiffness of the neck being quickly followed by rigidity in the muscles of the jaw, and this at once aroused our suspicions. There suspicions, brwerer, we must confess, were somewhat too easily allayen by thinking that we had to deal with an extremely rare manifestation-tetanoid spa-m, of that perennial pitfall. and simulator of diseave termed "hysteria." The trismus, however, was not sudden in onset and never became abulute, an haprens in hysteria; still the variation in the amount. of the trismu= seemed to point to functional rather than to organic disea-e. Due prominence was not at first 
given to the fact, as set down by Gowers, that "there is an absence of rigidity in the neck in hysteria," ${ }^{2}$ and Gowers's most sound and excellent advice, "that the symptoms of tetanus coming on after an injury or exposure to cold must not be treated lightly because they occur in a bysterical person or follow fear of the disease," 3 was not at first sufficiently acted on. 'Though not hysterical our patient was distinctly neurotic and both knew about and had an awful fear of "lockjaw."

We were loth, too, as is not surprising, to admit that the inoculation for vaccinia had at the same time sown the seeds of tetanus. Neither of us had ever heard of such a thing, yet we have been able to find references to 10 cases, where tetanus has occured after vaccination. Six, and perhaps seven, of these ${ }^{4}$ are reported from America and two from the West Indies, ${ }^{5}$ where tetanus itself is very common, but, unfortunately, we have not access to any of the journals containing the records of them. We learn, however, from the title of one paper that the patient was cured by large doses of opium; and from epitomes in THE LANCET and the British Medical Journal we get a very fair idea of the course of events in two fatal cases. A single and fatal instance of this accident is reported in Great Britain. ${ }^{6}$

In a case related by Toms ${ }^{7}$ "tetanus occurred 24 days after vaccination in a weakly girl, aged five and a half years. Bovine virus was used with all possible antiseptic precautions. On the eighteenth day the child was ill, the temperature was $100.5^{\circ} \mathrm{F}$., and the ulcer at the site of the vaccination was deep, slightly indurated, and discharging a sanious pus. These appearances improved under treatment and six days later the child had an aphthous stomatitis, and now for the first time, while the mouth was being examined, some trismus was noted. The muscular rigidity spread, opisthotonos developed, and the child died trom cedema of the lungs 35 days after the vaccination. At the time of death the vlcer on the arm appeared to be healthy. Against the theory that the tetanus was due to vaccination are (1) the presence of the sore mouth; (2) the length of the incubation period if counted from the vaccination; and, most important, (3) the inability to cultivate the tetanus bacillus from the wound of the arm. The possible sources of infection of the arm were (1) the vaseline, (2) the sponge, and (3) the unsterilised dressings used by the patient's friends,' but all there may perhaps be ignored in view of the bacteriologist's finding that the arm was not infected with tetanus bacilli, th ough it must be confessed that this finding loses much of its weight when we consider how very, very difficult it is to cultivate tetanus bacilli from wounds. The interval between the vaccination and the onset of trismus is some. what over the average, but by no means too long for the incubation period of tetanus.

In another case investigated by the Royal Commission on Vaccination," and published in their reports, "a female child, aged two months, developed trismus on the twentythird day after vaccination. The child's arm did well up to the ninth day, when it became inflamed. Suppuration but no erysipelas set in on the fourteenth day. The child died with opisthotonos and interference with respiration on the twenty-sixth day. The reporter acquits the lymph used and the operation of being the cause of tetanus which is most unusual after vaccination. This child was vaccinated from

${ }^{2}$ W. R. Gowers: A Manual of Diseases of the Nervous System 1893, vol, ii., p. 692

4 W. T. O. Bates: Traumatic Tetanus from Vaccination, Transactions of the South Carolina Medical Association, Charleston, 1882, vol. xxxii., p. 105. H. J. Berkeley : A Case of Tetanus following Vaceination. MaryTHE LANCET, Nov. 4th, 1882, p. 792. J B. Cottman: A Case of Tetanus Prorluced from Vaccination, Cured by Large Doses of Opium, New Orleans Medical and Surgical Journal, 1854-55, vol. xi., p. 783, T. Dimon: Tetanus following Vaccination; Importance of Reliable Virus, St. Louis Cour. Med., 1882, vol. vii., pp 310-312. G. Ross: Tetanus following Vaceination, South Clinic, Richmond, 1878-79 vol. i., p. 468 ; also Virginia Medical Monthly, Richmond, 18 . News, Philadelphia, Feb. 24th, 1894 ; also Brit. Men. Jour., in Eprtome of Current Medical Literature, April 28th, 1894, p. 65. Oxford, quoted ty Toms (date and place of communication not lpown)

by Toms (date and place of communication not known).

5 Garcia Rijo Rudesindo: Tetano Consecutivo á la Vacuna, Crón. Med.-quir. de la Habana, 1886, vol. xii., p. 388 . J. Rinz: Infección

p. 649 .

Papers relating to Cases in which Death or Non-fatal Injury was alleged ar suggested to have been caused by, or otherwise connected Commission on Vaccination. 1897, Case X., p. 6. Also THE LANCET, Commission on Vaccination.
Nov. 2nd, 1889, pp. 922 and 970.

Nov. 2nd, 1889, pp. 922 and 970.

8 Appendix IX., referred to in Note 5. the arm of a female infant, aged five months, and at the same time and with the same 'lymph' six other children were vaccinated, and none of them developed tetanus or sore arms. But tetanus may follow very slight injuries, and it is regarded as noteworthy that 28 days after the death of the above child another infant, aged 10 days, at a house half a mile off, died from tetanus supervening on an unhealed umbilicus."

In yet another case" "the patient was a healthy man, aged 40. Some days after the operation, which was performed about the middle of January last, he exposed himself to severe cold, and the arm became much swollen and the integument around the scab inflamed. No other lesion could be detected anywhere in the body. On Feb. 7th he noticed a little stiffness of the jaw and there was slight opisthotonos, accompanied on the following clay by profuse perspirations and muscular spasms of the lower extremities. These symptoms increased in severity till the 13th, on the evening of which day death took place, the pulse at noon having been 120 , the respiration 44 , and the temperature $102 \cdot 0^{\circ}$. Treatment throughout was by chloral hydrate and kalium bromide. Constipation was persistent during the entire illness. Dr. Berkeley acquits the virus of any share in producing the tetanus."

In our patient the first tetanic symptoms apreared exactly 12 dass after the vaccination and two days after exposure to a draught. "The interral between the infliction of the wound and the appearance of the first symptcms of tetanus is usually from five to 14 days."10 "Idiopathic tetanus usually follows exposure to cold, and when due to a single exposure, so that the interval could be ascertained, it has rarely exceeded two days and bas frequently been only a few hours. In many cases classed as traumatic tetanus there has been an exposure to cold after the receipt of the wound." 1 Now, considered from the point of view of length of incubation period we would be at a loss whether to ascribe the tetanic infection to the exposure to cold on the one hand or to the vaccination on the other, for both satisfy this criterion, were it not for the now generally accepted idea that all cases of tetanus are traumatic, and the further dictum of Gowers that "cases in which tetanus is apparently. excited by cold in a person who has had a trifling injury, perhaps some time before, must be included for scientific purposes in the traumatic variety." 12 "The influcnce of cold has been thought to aid the occurrence of puerperal and neonatal tetanus," "13 but on the whole it seems more in accordance with modern thought to regard "the exposure to cold as somehow or other predisposing the nel vous system to suffer from a minute dose of the tetanic poison." "That there could not have been a large dose introduced in our case is amply proved by the mildness of the symptoms, and we must conclude that the extra inritation inrolved in the exposure to cold simply played the part of firing the mine already laid, just as Verneuil has noted that surgical interference with a wound may apparently excite tetanus. And as bearing out the same idea is the case recorded by Terrier. "An injured toe had beccme gangrenous and was ampu. tated. Trismus, absent before, was present a quarter of an hour aftewards, and the patient averied that the pain of the operation distinctly made his jaw muscles contract while the amputation was being performed."1.5 In Berkeley's case there was also exposure to severe cold ir, the interval between the vaccination and the development of tetanic symptoms.

The incubation period in our case was shorter-by 12 days in one and by 11 in the other-than in two of the cases mentioned above, and there was no abrasion, ulceration, or injury of cutaneous or mucous surfaces to interfere with our calculations, as in the case reported by Toms. In all these reported. cases, however, the duration of incubation if counted from the time of the vaccination is well within the limits--viz., a month-as set down by Gowers. ${ }^{16}$

Now, granted that the poison of tetanus was introduced by the process of vaccination, we have the further question to answer-Whence came the poison? We do not see how the lymph can be blamed, for, though drawn from an. agricultural source, the calf, which at once suggests manure and dung heaps, several people were inoculated from the same tube, and this one patient alone developed tetanus. Further, the probability is that had the lymph been the infective agent the whole output of a certain date would

9 Berkeley, loc. cit.; THe Lancet, Nov. 4th, 1882, p. 792.

14 Ibid., p. 678.12 Ibid.. p. $692 . \quad$ is Ibid., p. $678 . \quad$ 14 Ibid. 
hare been contaminated, and a regular epidemic would have resulted, such as occurred in Italy and more recently in the City of St. Louis as the result of using diphtheria antitoxin. ${ }^{i}$ It is important, too, in this connexion, to note that in the case examined by the Royal Commission on Vaccination, human lymph was used, and only one child out of the seven vaccinated frum the same source and in the same manner developed tetanus. No more can we blame the lancet or the hands of the operator, for at that particular time (in the spring of 1901) medical men were doing little else but revaccinating, and yet out of the large number raccinated on that particular day only this one patient developed tetanus.

IVe must conclude, then, that the source of the infection was in the patient's own skin, and it is to be remembered. that the site of the inoculation was the leg, though in the three other cases referred to the arm was chosen. According to Thamhayn the order of frequency with which the several parts have been the seat of the wound that caused tetanus is (1) the hand, (2) the leg, (3) the foot, (4) the head and neck, and (5) the arm and trunk. ${ }^{1 *}$ of the many patients whom we vaccinated in this case alone did we do so on the leg, though we have seen it stated with authority that such is commonly practised on the infants of Canada, is the custom in Spain, and is unattended by danger. ${ }^{19}$ But babies differ very materially from adults, and it is superfluous to point out how much nearer in the latter the leg than the arm, say, is to the great source of infection, viz., the earth. Moreover, it is important in this relation to remark that our patient conformed to the prevalent custom of wearing a long dress, and it seems hard to understand how skirts trailing along our city streets can fail to sweep up material, composed of finely pulveri-ed earth, horse-dung, sc., and most likely containing tetanus bacilli and spores. The constant to-and-fro lap-flapping of the dress must cause the stockings very quickly to become seriously soiled with infective material, and in the case of women where widemeshed stockings alone afford covering for the nether limbs below the knee there is no difficulty in following the infective agent through the stockings on to the legs themselves. It seems to us that, let a woman be ever so cleanly in her habits, perform ablutions as of ten as she likes, and change her garments as frequently as she pleases, she has only to walk a few yards-if she trails her dress-to furnish the bacteriologist with more than food for rellection.

The antiseptic measures employed, though considerable, were of course quite inadequate to destroy any spores of the tetanus bacillus lodged about the glands or the pits of the skin; indeed, a few incantations would have been as effective. The very fact, too, that the operation of vaccination is designed to implant and retain the vaccine virus by means of numerous and almost bloodless punctures, proddings, and rubbings ensures that any other foreign matter present will be well introduced, and offers special facilities for growth to the bacilli and spores of tetanus. Tetanus is specially common after punctured and contused wounds, and what is the vaccination wound but a congeries of minute punctures and contusions? A surgical operation conld most likely have been carried out on this leg with impunity without any additional antiseptic precautions, for the clean incision of the surgeon's knife militates against the very conditions which the vaccinator's lancet fosters. We regret now that we covered up the vaccination mark at the beginning and are more than sorry that through a misapprehension our patient converted the meant-to-be-temporary protection into a permanent covering, for the somewhat impervious dressing may have helped to produce those anaerobic conditions so necessary for the development of the tetanus bacillus. Moreover, this sealing-up of the wound has the disadvantage that it quite puts out of court any possibility of infection of the wound subsequently to the vaccination. Further, it is important to note that in the three fatal cases which we have quoted all the patients had very sore arms, two of the arms being described as suppurating. In our own case, also, the vaccination mark, though not surrounded by induration and inflammation and though not associated with enlargement of glands, did not look particularly healthy, still that there was nothing much amiss was amply proved by the absence of abnormal temperature, and the fact of a single poultice at once converting it into

17 Tetanus and Diphtheria Antitoxin, Brit. Med. Jour., Jan. 11th, 1902, p. 104. a healthy healing wound. "Kitasato now holds that in the natural infection in man, along with tetanus spores, the presence of foreign material or of other bacteria is necessary. Spores alone or tetanus bacilli without spores die in the tissues, and tetanus does not result." 20 Thus we see how "bad arms" may help in the development of tetanus; nevertheless, it is perhaps not entirely a question of the successful or non-successful use of antiseptics, and it is quite in harmony with Kitasato's teaching to believe that the vaccine virus itself and the consequences which it produces are quite capable of injuring the tissues to such a degree that the tetanus spores or bacilli may become pathogenic without the presence of any streptococci or staphylococci to aid and abet them.

'This case was treated throughout by chloral hydrate, eight ounces three drachms and 10 grains being administered over a period of 44 days. No mild or heroic surgical measures were carried out and no antitoxin, as already mentioned, was employed. Had the tetanus antitoxin been used this case might have gone to swell the badly needed good results after such treatment. Encouraged by the confident prognosis of Professor Sir Hector Cameron, which was endorsed by Professor Monro, we waited (till not only our own patience but that of the patient's friends also was well-nigh exhausted) for the elaboration of a natural antitoxin by the patient's own tissues. That this consummation took a very long time we must confess, and it is possible that a resort to the use of animal antitoxin might have considerably expedited matters, though the ultimate result left nothing to be desired.

It may be asked what good end can possibly be served by reporting a case of this sort, so we hasten to make answer that we feel that it is only our duty to our medical brethren to do so; and if the perusal of this commurication impresses the reader as we have been impressed, more especially since we began to study the literature of vaccination, with the great necessity for the employment of adequate antiseptic precautions when performing this most trivial of operations, the great cause of vaccination will be considerably helped thereby. It is more than doubtful whether many of us use an antiseptic otherwise than as a fetich. What are adequate antiseptic precautions? Can the skin be rendered aseptic or safe by scrubbing for a few minutes with disinfectants? Most surgeons, we believe, would give a very decided negative to the latter question and yet vaccination is riskier in some ways than a clean cutting operation. We are disappointed on reading over the third schedule of the Vaccination Order of Oct. 18th, 1898, issued by the Local Government Board, England, to find that though considerable space is devoted to the sterilisation of instruments, and the care and use of lymph, there is nothing more definite as regards the preparation of the skin than, "Vaccination should at every stage be carried out with aseptic precautions, and these should include: First, the cleansing of the surface of the skin before vaccination." ${ }_{2}$ The neglect of the skin and idolatry of sterilised instruments are reflected likewise in the advertisement columns of the medical journals where instrument-makers are seen to vie with one another as to who shall produce the daintiest and most easily sterilisable outfit.

The accident in our case, fortunately so very rare, could hardly, we think, have been foreseen, and possibly could only have been prevented by adopting the same elaborate measures (extending over days) to render the skin aseptic which the surgeon employs before performing a major operation.

Since the above conclusions were formed and set down it has come to our notice that some cases of tetanus (number not stated) have occurred after vaccination in Camden, New Jersey, and we also learn that a committee has inquired into and reported on the matter. ${ }^{22}$ We have tried to apply the findings of this committee of experts to our own case, and though we recognise that any opinion coming with such high authority cannot be treated lightly we see no reason to amend our conclusions in the slightest. "In every case acute tetanus occurred in from three to four weeks after vaccination, whereas if the virus had been contaminated tetanus would have ensued within nine days after vaccination. A further proof of the purity of the virus is afforded by the negative results obtained from innculation experiments on

20 Muir and Ritchie : Manual of Bacteriology, 1899, p. 389.

22 Brit. Med. Jour., Feb. Ist, 1902 , p. 285 ; St. Paul Medical Journal 
animals with samples of the different lymphs used." We do not know what authority the committee bas for saying that if the virus had been contaminated tetanus would have ensued within nine days after vaccination. The authorities whom we have consulted do not give such a limited incubation period for tetanus. We believe that the lymph used by us was as free from tetanic infection as was that employed by the Camdenites, but our faith in our explanation by skin infection at the time of the vaccination remains unshaken. "In every instance it was found that vaccination had been performed in a correct and cleanly manner." But this has been said about all the cases of tetanus supervening on vaccination that we have read of and might be also truthfully said about our own case. "The committee thinks that the tetanus cases in Camden are to be explained by atmospheric and telluric conditions. There had been a long period of dry weather, with high winds, so that tetanus germs, which have their nornial habitat in the earth, dust, dirt of stables, scc., were constantly distributed in the atmosphere. One case of tetanus occurred from gunshot wound during the same period in a boy who had not been vaccinated-a fact which the committee regard as proving that the tetanus germs were in the atmosphere." Now, in all seriousness we would ask, Is it sound reasoning to conclude that because one of the many injured and wounded persons developed tetanus during the same period that several cases of tetanus were occurring after vaccination, that the tetanus germs must have been in the air? Of course, the tetanus germs were in the air-they are always there more or less; but is not the logical conclusion to be derived from these facts what we have already stated--viz., that the vaccination wound offers special facilities for growth to the tetanus bacillus? If atmospheric and telluric conditions alone were to blame vaccination would not have claimed cases while injuries claimed but one case. "In all the cases it was found on careful examination that the wound had been exposed by the scab being knocked off or removed or the arm had been injured and infection resulted; frequently children scratched the vaccinated area with their dirty fingers and nails and thus infected the wound. It is given as the unanimous opinion of the Board of Health, as well as of its committee of experts, that tetanus or any other infection can never occur if the vaccination is properly protected from contact with the atmosphere or with soiled clothing, bandages, \&c." In our case, as already mentioned, the scab was not removed till the symptoms of tetanus developed, and even had our patient been in possession of dirty fingers and nails she could not have scratched the wound on account of the covering that was over it. Proper protection after vaccination is not everything, and in all humility we would direct the attention of the Camden Health Board to the skin of its patients as the not improbable source of the infectic $n$.

\section{NOTES OF 15 CASES OF OPERATION FOR INTERNAL DERANGEMENT OF THE KNEE-JOINT.}

BY J. M. COTTERILL, F.R.C.S. EDIN.,

SURGEON TO THE EDINBURGH ROYAL INFIRMARY ; LECTURYR OY CLINICAI SURGERY IN THE EDINBURGH SCHOOL OF MEDICINE.

JUDGING from the views expressed in various text-books and recent papers on the above condition there still seems to be a considerable diversity of opinion with regard to many points in the etiology, pathology, and treatment of so-called dislocation of the semilunar cartilage. It may not be inopportune, therefore, to add the experience gathered from 15 cases of operation for this injury, as well as impressions formed from the observation of a large number of cases in which operation was not considered necessary.

The few points which I propose to discuss naturally fall under the following heads: (1) etiology; (2) pathology ; (3) symptoms and diagnosis; and (4) treatment.

\section{ETIOLOGY.}

Most writers are fairly agreed on this point. The accident usually happens when the knee is partly flexed by a sudden external twist of the tibia on the femur, or, perhaps, more strictly speaking, an internal twist of the femur on the tibia. Such a thing may happen when a person steps out of a moving conveyance at too great an angle to the line of its movement, when he rises suddenly from the kneeling position, or in the course of football or similar games. I have known the internal cartilage to be displaced in three cases by a boy falling on the outer aspect of the patient's extended leg in a "maul" at football, and another case was caused by a vigorous kick at football missing the ball. I have not met with a case where a direct blow applied to the cartilage was the cause of its displacement, but I have known an accident of this kind to account for the separation of a chip of the bone (the tibia) with articular cartilage attached, this condition being found on opening the joint. As the cartilage recedes considerably into the joint on flexion and is so well protected by the adjacent margins of the tibia and femur in extension this cause for dislocation must be very rare, though it is said to occur. There can be no doubt that, as Mr. Annandale noted some time ago, miners and others who habitually kneel at their work are particularly prone to "derangement" of the knee. I have seen several cases in such people. Chronic synovitis is also recognised as a predisposing cause. It is a matter of common experience in cases where the cartilage has been previously dislocated and where satisfactory new adhesions have not formed, that the least twist of the knee, a slight tap on the inner side of the foot, and similar trivial causes will readily reproduce the displacement.

With regard to the relative frequency with which the internal or external cartilage is affected, most writers are now agreed that the former is very much more commonly at fault than the latter. I do not know whether Mr. R. J. Godlee still adheres to the contrary opinion, but this viewan incorrect one, as I venture to believe-is still held by some authorities, and apptars, for instance, in the last edition of Da Costa's "Modern Surgery." My own experience coincides. with that of Mr. Annandale, Mr. A. E. J. Barker, and others. Out of the 15 cases oferated upon only one has been external, and I have only seen two other "external" ca: es in some 50 cases observed but not operated upon. In every one of the 15 operation cases the displacement of the cartilage (or of part of it) was inwards towards the centre of the joint, and while now and then one sees a case oi external or marginal displacement I believe the condition to be quite exceptional.

Cases have frequently been noted in which there was tearing of the cartilage. Mr. Barker, ${ }^{1}$ in an interesting paper, describes various kinds of longitudinal splitting which may take place. The accompanying illustration (Fig. 1) is an excellent example of what he terms " central splitting," the cartilage being split from end to end, the separated strip of the meniscus having found its way into the middle of the joint and lying against the crucial ligament.

Separation of the anterior attachment of the cartilage to the tibia is of common occurrence. I have met with it in several cases. Mr. Barker states that in none of his cares was the cartilage torn across at any point between its two ends. Strangely enough I have met with this condition in almost half of my operations, and so common a circumstance does it thus appear to me to be that I think that the term "dislocation of the semilunar cartilage" is very often an incorrect one, and prefer Hey's old name of " internal derangement of the knee-joint," which, if somewhat vague, is at least not absolutely misleading. Out of my 15 operation cases six have presented a distinct transverse tear through the edge of the meniscus, the torn fragment projecting into the joint and causing the chararteristic locking, while the greater part of the cartilage remained in most cases fixed in its normal position. (Vide Figs. 2, 3 , 4, and 5.) Fig. 6 represents the only case of external cartilage removed, and here there seems to have been some longitudinal splitting, causing thinning of the meniscus towards its middle. In one old-standing case the cartilage was found to be greatly thickened; in another the "ligamentum alare" and "infra-patellar pad of fat" were found to be much thickened and had to be removed in part alorig with the torn cartilage.

\section{SYMPTOMS AND DIAGNOSIS.}

The essential point in diagnosis is the inability fully to extend the knee. It is often stated that the joint is locked and that movements not only of extension but of flexion are impossible. Now, while a patient instinctively holds any 